\title{
Acceso al suelo y desarrollo urbano en el periurbano
}

\author{
Access to the soil and urban development in the periurbano \\ platense
}

Andrea Di Croce Garay andreadicroce@hotmail.com http://orcid.org/0000-0002-7173-6555

Centro Interdisciplinario De Estudios Complejos; Facultad de Arquitectura y Urbanismo; Universidad Nacional de La Plata (Argentina)

\section{Resumen}

En este trabajo presentaremos una comparación de tres experiencias de producción urbana en el periurbano platense, cada una de ellas propia de un sector social: barrios cerrados, PROCREAR y asentamientos populares informales (API). Para ello se indagará en las características que tomaron los procesos de acceso al suelo y desarrollo urbano, y la localización geográfica que asume cada experiencia.

Para poder desarrollar el trabajo se utilizaron fuentes bibliográficas, así como entrevistas propias. 
Los primeros análisis muestran características propias bien definidas para cada caso, distintos tipos de vínculos con los Estados, y resultados urbanos marcadamente diferentes entre sí, lo que corrobora la idea de que la producción urbana y la transformación territorial están fuertemente atravesadas por las características de la clase social que las está llevando adelante.

Palabras clave: Periurbano platense; acceso al suelo; desarrollo urbano; clases sociales.

Abstract

In this paper we will present a comparison of three experiences of urban production in the platani peri-urban, each one of them belonging to a social sector: closed neighborhoods, PROCREAR and informal popular settlements (API). To do this, we will investigate the characteristics that the processes of access to land and urban development took, and the geographical location that each experience assumes.

In order to develop the work, bibliographical sources were used, as well as own interviews.

The first analyzes show well-defined characteristics for each case, different types of links with the States, and markedly different urban results, which corroborates the idea that urban production and territorial transformation are strongly affected by the characteristics of the social class that is leading them forward.

Keywords: Platanian periurban; access to land; urban development; social classes.

La ciudad de La Plata es reconocida por la planificación de su casco histórico, su famoso "cuadrado y diagonales". Sin embargo, el territorio que conforma el Partido abarca una amplia heterogeneidad en su conformación social, y sus formas de ocupar y producir el territorio. Su periurbano se fue ocupando y transformando desde la fundación de la ciudad con características heterogéneas, con un crecimiento acelerado en las últimas décadas. Dentro de ese crecimiento, pueden reconocerse experiencias que responden a las tres lógicas de producción urbana desarrolladas por Abramo (2012: 36): la lógica privada, la estatal y la de la necesidad; cada una de las cuales presentan características diferenciadas sobre los procesos de desarrollo urbano, y sobre el funcionamiento del mercado de suelo. 
Para el desarrollo de este trabajo se indagará en tres procesos bien diferenciados, desarrolladas durante los últimos años (cada uno en distintos recortes temporales (1), que dieron lugar a la producción urbana de forma difusa (Ídem) en el periurbano de este Partido, cada uno de los cuales responde a una de las tres mencionadas lógicas:

-La producción de barrios desarrollados a partir del programa Pro.Cre.Ar (lógica estatal). -La producción de barrios cerrados (lógica privada).

-Producción desde la informalidad: tomas de tierras, villas y asentamientos (lógica de la necesidad).

Si bien en la ocupación periurbana de La Plata se desarrollan otros procesos de ocupación residencial (principalmente procesos desarrollados por productores hortícolas, y las construcciones privadas pertenecientes mayormente a sectores medios de la población), se define desarrollar los ejemplos mencionados por ser de un impacto relevante a nivel local, y por ser experiencias explícitamente diferenciadas entre sí, con distintos puntos de partida y de llegada.

Para desarrollar el análisis comparativo, se proponen tres ejes de indagación:

-¿Cuál es la magnitud de cada experiencia, en términos de superficie que cada una requirió? Envergadura a nivel local.

- ¿Cómo fue el proceso de acceso a las tierras en cada caso? Características de los tipos de mercado de suelos involucrados, cuáles son las características de las tierras involucradas (sus valores, requisitos para acceder, accesibilidad a infraestructura y servicios); a qué tierras accede cada sector social.

- ¿Cómo se llevó adelante el proceso de desarrollo urbano en cada proceso? ¿Hubo procesos de organización para acceder a la urbanización? ¿Intervino o interviene el Estado? ¿Cómo? ¿Hubo autogestión en casos de falta de propuestas estatales? ¿Cómo afectaron estas experiencias a la estructura urbana local?

Para ese análisis, es de relevancia mencionar antes aspectos teóricos que aportarán al trabajo. El proceso de urbanización argentino, aunque de grandes similitudes al latinoamericano, posee algunas particularidades propias. De esas particularidades tomaremos como una de las más relevantes la existencia de una clase media que no es de tan fácil reconocimiento en los demás países del territorio latinoamericano. Esta peculiaridad se refleja en la conformación del territorio, en los procesos de ocupación territorial que cada sector desarrolla. 
En nuestro país, después de décadas de migraciones internas el $90 \%$ de población es urbana. A partir de los años 80 comienza un fuerte crecimiento de ocupación de las periferias por vías informales (villas y asentamientos que aunque ya existían desde hacía años, soportan una oleada de nuevos fenómenos políticos, sociales, económicos, urbanísticos post-dictadura; y un auge de barrios cerrados en los 90). Estas periferias alojaban hasta el momento procesos de urbanización de menor escala, algunos de los que incluían programas estatales de vivienda para clases medias y populares (Yujnovsky, 1984).

Estos procesos de periurbanización están condicionados por lo que Del Río (2012: 177) denomina mercado de localizaciones residenciales intraurbanas, donde la configuración espacial, la calidad urbana y el perfil socioeconómico desarrollan relaciones intrínsecas. Podremos ver en los análisis cómo se dan estas relaciones, ligadas a lo que Jaramillo (2003: 16) denomina Renta Monopólica de Segregación, vinculando el acceso a determinados mercados de suelos, diferenciado por clases. Adherimos en este punto a lo que ese autor desarrolla sobre la paradoja de que la tierra tenga valor, partiendo de que la apropiación y la necesidad de acceder al bien es lo que da valor a la tierra.

Utilizando estas herramientas teóricas, se propone abordar los casos de estudio y analizar sus lógicas de desarrollo urbano y acceso al suelo.

\section{Presentación de casos}

Antes de realizar un análisis de los casos, presentaremos a cada uno de ellos a fines de poder conocerlos desde sus características generales para luego profundizar desde el análisis comparativo. Para esta primera presentación intentaremos recorrer las características que hacen a los procesos de cada caso, las composiciones sociales de quienes los protagonizan, y a las reconstrucciones genealógicas.

Así como describe Frediani:

la expansión urbana residencial hacia la periferia se traduce en una competencia por el territorio entre diferentes usos del suelo y actividades: aumento de la ocupación de nuevas superficies en los límites físicos de la ciudad y retracción de suelo rural. Así, los suelos agrícola-intensivos van cediendo lugar a una ocupación relacionada con el uso residencial permanente o de fin de semana-, el uso recreativo o de esparcimiento, el uso industrial, entre otros (2009: 8). 
Esta competencia, podemos agregar, no se da únicamente entre los posibles usos (rural o urbano), sino también entre sectores sociales que, dependiendo de sus posibilidades económicas, acceden a determinados sectores de los bordes urbanos. En ese sentido, podemos decir que si bien las periferias son ocupadas por diversos sectores sociales, son distintos los factores que hacen a que cada uno se instale en cada zona: por un lado, los factores culturales,

\begin{abstract}
representado por un sector de clase media y media-alta, que busca alejarse del centro por propia elección, dando prioridad a la calidad de vida antes que a la proximidad al mismo. Estos sectores migran fundamentalmente hacia el N-NO del Partido. El segundo se centra en la repercusión que tuvo la crisis económica que se inició a mediados de los '70, y se profundizó en los ' 80 y fines de los ' 90 , y que en nuestra región determinó la prolongación de la ciudad hacia el sur; se destaca el caso de Villa Elvira, adonde arribó en los últimos años un gran número de migrantes intraprovinciales y extranjeros. Este éxodo hacia el sur parece estar motorizado económicamente por el cuentapropismo (Frediani, 2009: 9).
\end{abstract}

A partir de ello podemos afirmar que en la construcción de la periferia platense se asientan sectores de ingresos altos, medios y bajos, desarrollando diversas estrategias de selección de las tierras, acceso al suelo, urbanización, las cuales intentaremos abordar.

\title{
PROCREAR
}

El programa PROCREAR fue lanzado el 12 de junio de 2012 y preveía entregar 400 mil créditos entre 2012 y 2015. El programa se conformaba por las siguientes líneas:

\section{Construcción}

2. Ampliación y terminación

3. Refacción

4. Compra de terreno y construcción

5. Compra de vivienda a estrenar

6. En algunos lugares del país se han concretado Desarrollos Urbanísticos a través de la cesión de tierras fiscales.

Como señala Ventura, 
es importante destacar, para una lectura más anclada del proceso, que el Pro.Cre.Ar además de favorecer el acceso a la vivienda, ha sido un importante impulso a la industria de la construcción, a la producción en general, así como un gran generador de empleo. Estos componentes lo colocan en un lugar de relevancia nacional que excede su carácter específico de plan de vivienda, y adquiere una importancia vinculada a la economía toda (2015: 6)

A fines de analizar el impacto urbano de este programa, tomaremos en cuenta las experiencias surgidas de la línea 4: por un lado las líneas 2, 3 y 5 no implican necesariamente construcción de superficie nueva; mientras que sobre la línea 1 -aunque implica construcción de superficieno se ha podido acceder a datos que permitan una caracterización exhaustiva, ligado a que son construcciones individuales que las familias ya poseían el lote o accedieron a él por vías individuales. Por otro lado, para la línea 6, si bien en el sitio web de ProCreAr figuran dos loteos aprobados, no fueron aún utilizados.

La línea 4 dio inicio a una serie de inconvenientes ligados a los aumentos de precios de suelo, producto de procesos de especulación inmobiliaria. Como respuesta se lanzó en La Plata el Programa de Generación de Suelo Urbano (Progesur), con el que se apuntó a conseguir lotes que se vendieran a no más de 150000 pesos, valor máximo fijado por el Programa. Por otro lado -y con organización de los y las beneficiarios/as mediante- se aprobó la Ordenanza 11094/13, que determinó la puesta en marcha de tres vías (1, 2 y 3) para la creación de suelo urbano:

-Vía 1: consistió en la apertura de un registro de beneficiarios/as y otro de oferentes de tierras. En este caso los/as propietarios/as de tierras rurales podían ofertar sus tierras al Municipio, para que sean rezonificadas y urbanizadas. Los nuevos lotes se pusieron a disposición exclusivamente de beneficiarios/as del Pro.Cre.Ar que previamente debieron anotarse en el registro.

-Vía 2: promovió la búsqueda de tierras rurales por parte de los/las beneficiarios/as del Programa, quienes debían realizar acuerdos con los/las propietarios/as, y presentar el plan de subdivisión. Las tierras ingresaban al Registro de Oferentes de la vía 1, pero acompañadas con los compromisos de compra-venta firmados entre las partes.

-Vía 3: así como describe Ventura (2015:15), se prevé que la ordenanza y sus alcances podrán ser extendidos a favor de quienes -a la fecha en que ésta fue sancionada- sean titulares de fracciones indivisas, en la medida en que garanticen la provisión de infraestructura básica. 
Como resultado, se realizaron rezonificaciones en parcelas rurales de Parque Sicardi, Altos de San Lorenzo, Olmos, Melchor Romero, Villa Elvira, Arana, Abasto, Arturo Seguí y Los Hornos. Estas rezonificaciones dieron lugar a 11 nuevos barrios, en zonas generalmente desprovistas de servicios e infraestructura básicos, lo que ocasionó nuevos inconvenientes que fueron encontrando diversas respuestas en cada caso.

Los análisis revisados sobre estos barrios, así como los requisitos socioeconómicos que el Programa definía (principalmente la obligatoriedad de poseer un trabajo formal, y el límite salarial establecido para lo/las beneficiarios/as) permiten afirmar que fue un política destinada a los sectores medios y medios altos de la población, quienes tiene posibilidades de ahorro, de adquisición de otro crédito (para los casos en que el monto otorgado por ProCreAr no fue suficiente para finalizar la obra), de tener previamente un terreno adquirido por vías formales. Esta caracterización del grupo social será relevante a la hora de analizar los procesos de desarrollos urbanos de los barrios, teniendo en cuenta el capital social con que este sector cuenta.

\section{Barrios cerrados}

La legislación de la provincia de Buenos Aires reconoce dos figuras de urbanizaciones cerradas: clubes de campo y barrios cerrados. Estas tipologías tuvieron un fuerte impulso durante los años 90 en Argentina, en tierras periféricas a las ciudades, pero con buena conectividad. Se caracterizan por su uso residencial de baja densidad, con un alto porcentaje de superficie destinado al uso recreativo, equipamiento social y deportivo común.

Las urbanizaciones cerradas son reflejo de un periodo histórico caracterizado por la profundización de la brecha social, una de cuyas expresiones territoriales son los barrios de perímetros cerrados, con reglamentos que restringen la posibilidad de integrar estas urbanizaciones. Las familias nucleadas pertenecen a un mismo sector social de ingresos altos, las cuales definen juntarse a partir de discursos ligados a la búsqueda de una mejora en la calidad de vida, "superadora de la que ofrece la metrópoli, desde el punto de vista socioespacial y ambiental: mayor seguridad urbana, más espacio verde y contacto con la naturaleza, más y mejores opciones de esparcimiento y recreación" (Frediani, 2010: 84).

En el caso de La Plata, estos barrios se localizan principalmente en la zona noroeste y sudoeste del Partido, cercanos a vías rápidas de comunicación (Ruta 2, caminos Belgrano y Centenario). Es llamativa la escasez sobre antecedentes de investigaciones e informes sobre estos tipos de urbanizaciones: la mayor producción encontrada es la realizada por Frediani 
(2009), cuya actualización cuantitativa más reciente es de 2010. En esos trabajos, particularmente en su tesis doctoral, se contabilizaban 21 barrios cerrados, ubicados sobre 641 hectáreas (has.) (es decir, el $0.69 \%$ de la superficie total del Partido). Este registro le permitía afirmar que "el impacto generado por este tipo de emprendimientos urbanísticos en el Partido, no sería hasta el momento significativo".

Desde la aprobación del Código de Ordenamiento Urbano local en 2009, se aprobaron nuevas zonas para este tipo de emprendimientos. Como resultado de ello, y ante nuevos procesos de fragmentación socio espacial, hubo un crecimiento exponencial de estas tipologías. Mediante relevamientos y producción de información primaria (2), se pudieron reconocer al momento 35 barrios cerrados (Tabla 1), a los que se agregan 26 que están ya publicitados en venta (Tabla 2). Para este cálculo se contabilizaron los barrios cerrados, countries, y barrios que figuran como "loteos abiertos" pero que reúnen características similares a los barrios cerrados en tanto núcleos de familias de clase media alta que deciden vivir alejadas del centro en búsqueda de aparente tranquilidad. Es relevante mencionar la dificultad presentada a la hora de producir información sobre estas urbanizaciones, ante la escasez de información accesible públicamente y lo dificultoso en el acceso a los predios y a sus habitantes, lo que restringió la posibilidad de profundizar en el apartado de análisis comparativo. Es propuesta a futuro poder avanzar en los datos que para este trabajo no pudieron obtenerse, y propuesta para este trabajo analizar aquello que sea posible con los datos producidos al momento. 
Tabla 1. Barrios cerrados construidos en La Plata.

\section{BARRIOS CERRADOS CONSTRUIDOS EN LA PLATA}

\begin{tabular}{|c|c|c|}
\hline & BARRIO & SUP.(Ha) \\
\hline 1 & Aires Nuevos & 2 \\
\hline 2 & Altos de San José & 7 \\
\hline 3 & Altos del Golf & 2 \\
\hline 4 & Bo.Parque La Lula & 4 \\
\hline 5 & Don Luis & 4 \\
\hline 6 & El Cedro & 3 \\
\hline 7 & El Quimilar & 1 \\
\hline 8 & El Solar el Aguador & 17 \\
\hline 9 & Elida III & 1 \\
\hline 10 & Elida IV & 1 \\
\hline 11 & Fincas de Victoria & 11 \\
\hline 12 & Fincas del Duggan & 11 \\
\hline 13 & Gonnet I & 1 \\
\hline 14 & Grand Bell I y II & 130 \\
\hline 15 & Haras del Sur I & 114 \\
\hline 16 & La Araucaria 55 & 3 \\
\hline 17 & La Arbolada & 10 \\
\hline
\end{tabular}

\section{Elaboración propia}

\begin{tabular}{|c|c|c|}
\hline & BARRIO & SUP.(Ha) \\
\hline 18 & La Cándida & 32 \\
\hline 19 & La Elisa & 4 \\
\hline 20 & La Emilia & 28 \\
\hline 21 & La Torre & 7 \\
\hline 22 & Las Calandrias & 25 \\
\hline 23 & Las Cañitas & 3 \\
\hline 24 & Las muñecas I y II & 5 \\
\hline 25 & Lomas de City Bell & 80 \\
\hline 26 & Los Ceibos & 37 \\
\hline 27 & Prados de la Vega & 22 \\
\hline 28 & San facundo & 19 \\
\hline 29 & San Joaquín & 4 \\
\hline 30 & San Jorge & 1 \\
\hline 31 & $\begin{array}{l}\text { Santa Maria de La } \\
\text { Plata }\end{array}$ & 80 \\
\hline 32 & Solar del Parque & 1 \\
\hline 33 & Solares II & 3 \\
\hline 34 & Swan & 4 \\
\hline \multirow[t]{2}{*}{35} & Villas Las Marias & 3 \\
\hline & SUP. TOTA & 680 \\
\hline
\end{tabular}


Tabla 2. Barrios cerrados que ya están loteados, vendidos o publicitados.

BARRIOS CERRADOS PUBLICADOS, EN VENTA O VENDIDOS LOS LOTES

\begin{tabular}{|c|c|c|c|c|c|}
\hline & BARRIO & SUP.(Ha) & & BARRIO & SUP.(Ha) \\
\hline 1 & \multirow{2}{*}{$\begin{array}{l}\text { Altos del Plata } \\
\text { Haras del Sur IV }\end{array}$} & 4 & 14 & El Fresno & 3 \\
\hline 2 & & 1 & 15 & San Benito & 3 \\
\hline 3 & \multicolumn{2}{|l|}{ Campos de La Enriqueta } & 16 & $\begin{array}{l}\text { Barrio } \\
\text { Angosto }\end{array}$ & 4 \\
\hline 4 & Buona Terra & 2 & 17 & La Paulina & 35 \\
\hline 5 & Arana y 140 & 3 & 18 & La Cantera & 1 \\
\hline 6 & San Efren & 12 & 19 & La juanita & 1 \\
\hline 7 & El Cauce & 7 & 20 & $\begin{array}{l}\text { El } \\
\text { Cauquén }\end{array}$ & 3 \\
\hline 8 & Las Huertas & 3 & 21 & La Cañada & 60 \\
\hline 9 & San Joaquín & 9 & 22 & Pastizales & 17 \\
\hline 10 & Quintas de Mitre & 4 & 23 & $\begin{array}{l}\text { Cava } \\
\text { Pampa } \\
\text { Club de } \\
\text { Viña }\end{array}$ & 84 \\
\hline 11 & La Clementina & 4 & 24 & La Victoria & 100 \\
\hline 12 & Las Carolinas & 11 & 25 & La Florida I & 81 \\
\hline \multirow[t]{2}{*}{13} & \multirow[t]{2}{*}{ El Roble } & 3 & 26 & Miralagos & 174 \\
\hline & & & & SUP. TOTAL & 629 \\
\hline
\end{tabular}

Fuente: elaboración propia

Tomas de tierras, villas y asentamientos

La historia de la informalidad urbana platense nace junto con la construcción de la ciudad, con la formación de asentamientos ocupados por los/las trabajadores/as que ejecutaron la obra. Pero este fenómeno se fue profundizando con los años. En la etapa que comprende los años 1940-1970, caracterizada por una producción apuntada a la sustitución de importaciones, se desarrolla un importante proceso de suburbanización: caracterizado por el crecimiento 
industrial y los flujos migratorios internos, comienzan a ocuparse tierras periféricas mediante el mercado de loteos populares, mercado consumido por los trabajadores de esas industrias. Estos procesos, sin embargo, no tomarán una escala tan relevante como la que se dará en otras capitales -o en el caso local, con la llegada del neoliberalismo- sino que se mantendrán en el orden de fenómenos aislados.

Tras el Golpe contrarrevolucionario de 1976, se dio inicio a un periodo económico caracterizado por la desindustrialización, una fuerte caída del salario real, el deterioro de las condiciones laborales, la privatización de servicios y espacios públicos, y la disminución en gasto social (González, 2009: 5 y del Cueto, 2008: 18). Los cambios en los procesos económicos derivaron en cambios sociales, ligados a la fragmentación y exclusión social, así como a fuertes procesos de desigualdad, informalidad y empobrecimiento masivo. Estos nuevos escenarios fueron moldeando nuevas formas de (des)regulación urbana, conducida por el "libre" mercado, que condujeron a un aumento de la producción urbana desarrollada desde la lógica de la necesidad. Ante estos cambios socioeconómicos -y algunos otros normativos, como la aprobación de la Ley de ordenamiento del suelo 8912-, durante las últimas cuatro décadas se desarrollaron nuevas estrategias de acceso al suelo urbano por parte de los sectores populares, principalmente las tomas de tierras y el mercado informal del suelo. Estas estrategias tendrán su correlato en la conformación del territorio local, y las relaciones que en él se desarrollan.

En diciembre de 2015 se dio a conocer el Registro Público Provincial de Villas y Asentamientos Precarios (RPPVAP) (2015), desarrollado en el marco de la Ley Provincial de Acceso Justo al Hábitat 14449. Este Registro es el primero realizado oficialmente sobre esta temática, y permite visualizar (Tabla 3) rápidamente el fuerte crecimiento de producción urbana informal desarrollado en las últimas cuatro décadas (en este caso en la ciudad de La Plata, pero es un dato corroborable a nivel provincial): 
Tabla 3. Cantidad de villas, asentamientos y otros por década en el partido de La Plata, período 1960-2015.

\begin{tabular}{l|c|ccc|}
\hline Año & Cant. Villas & Cant. Asentamientos & Cant. Otros & Total V, AyO \\
\hline $1960-1989$ & 8 & 7 & 2 & 17 \\
\hline $1990-1999$ & 6 & 30 & 5 & 41 \\
$2000-2009$ & 9 & 32 & 2 & 43 \\
$2010-2015$ & 3 & 17 & 1 & 21 \\
TOTALES & 29 & 89 & 11 & 129 \\
\hline
\end{tabular}

Fuente: elaboración propia.

En primer lugar, podemos observar que durante las décadas del 90 y del 2000 se produjeron prácticamente la misma cantidad de barrios informales; y que el patrón es equivalente en lo registrado en el quinquenio 2010-2015. Es notorio también que en esos 25 años se produjo ciudad informal en un 600\% (112 villas o asentamientos) más que durante los 30 años del periodo 1960-1989 (17 villas o asentamientos).

La mayoría de los/las habitantes de estos barrios son migrantes internos y de países limítrofes, que llegan a la ciudad a partir de búsquedas laborales. Su condición de migrantes los hace parte de un circuito de informalidades retroalimentado en sí mismo: las dificultades para acceder a trabajos formales se expresa en la informalidad urbana, de salud, de acceso a la educación, etcétera.

Si bien estos datos cuantitativos son una expresión visible de la informalidad urbana, existe una parte invisibilizada: los intentos de ocupaciones de tierras. Desde un equipo de trabajo de la FAU UNLP (3) se encuentra en proceso un registro de estos intentos, independientemente de su desenlace. Ese registro contabilizó hasta el momento para el periodo 2000-2016 un total de 200 intentos de ocupaciones de tierras (periodo en el que el RPPVAP contabiliza 64 Villas o Asentamientos producidos). 


\section{Análisis comparativo}

A continuación desarrollaremos un análisis comparativo de los tres casos, a partir de tres ejes: la magnitud o envergadura a nivel local de cada experiencia, características de los procesos de acceso a las tierras, y características de los procesos de desarrollo urbano.

En primer lugar veamos el impacto de cada proceso analizando sus localizaciones y las superficies que abarcan. Utilizando la información recabada y producida sobre los tres tipos de producciones urbanas en cuestión, se desarrolló el mapeo de los barrios. Esta tarea tuvo como objetivos visualizar los sectores donde cada grupo de experiencias están mayormente emplazados, dimensionar la magnitud (en términos de cantidad de superficie), y a partir de ello el impacto que pudo haber generado en términos sociales y urbanísticos (Mapa 1).

A simple vista, es fácilmente legible que la mayoría de los barrios cerrados se localizan en el eje noroeste y sobre la Ruta 2, la producción urbana informal sobre el eje sureste, y las experiencias de Pro.Cre.Ar se ubican de forma dispersa. Es interesante mencionar que el análisis de las superficies permisadas a nivel local muestran cuantitativamente la producción formal por zonas en el Partido, registrándose las mayores cantidades en el casco urbano y eje noroeste, y las menores cantidades (exponencialmente menores) sobre el eje sudeste. Sin embargo, al comparar esta información con el crecimiento de la mancha urbana en cada eje, es notorio el crecimiento del eje sudeste (de menor cantidad de superficie permisada), lo que denota la producción de ciudad informal de esa zona. 
Mapa 1. Mapeo de barrios cerrados construidos y aprobados, barrios surgidos de la Via 2 del PROCREAR, e intentos de tomas de tierras en La Plata.

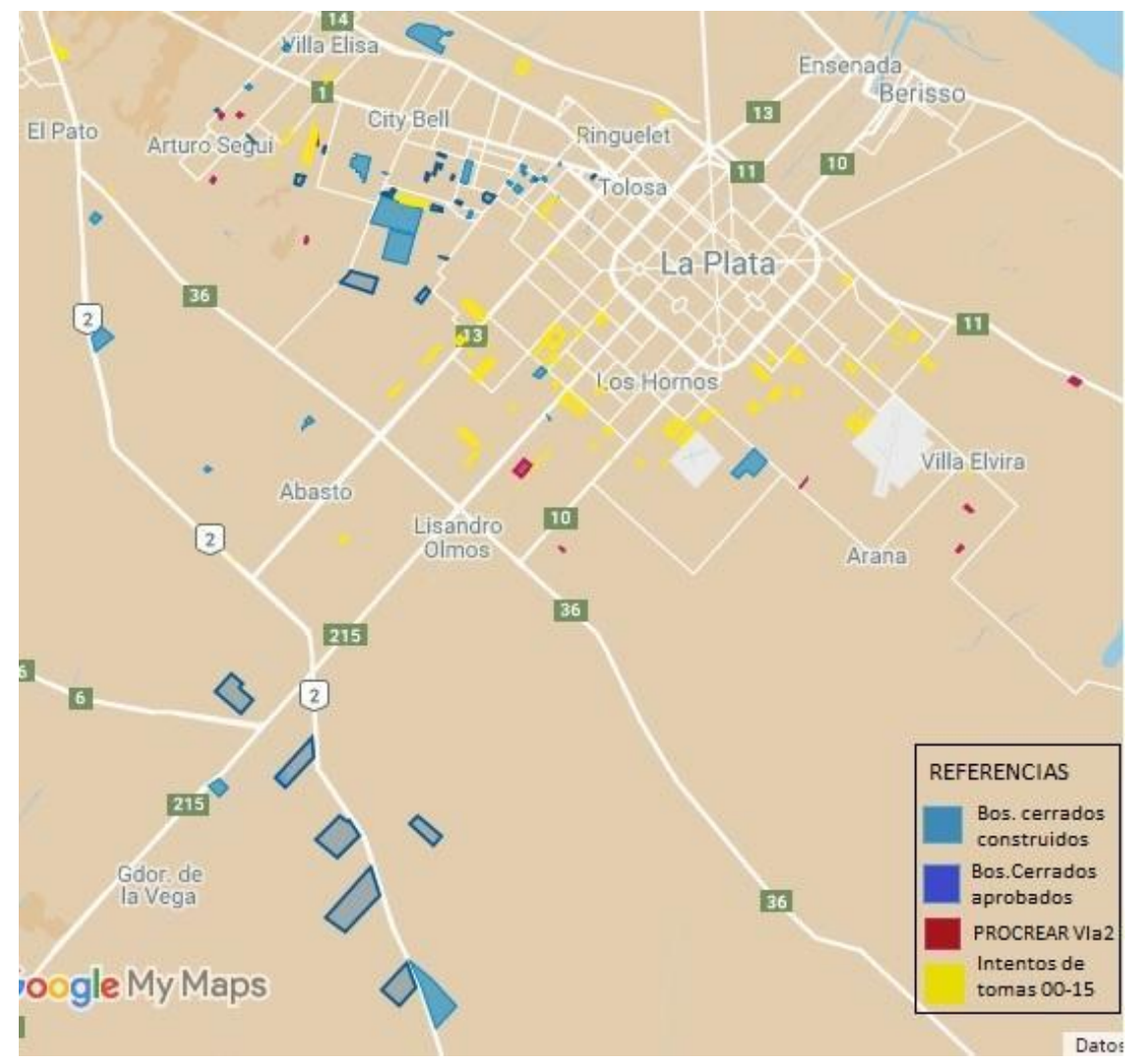

Elaboración propia a partir de registros propios.

Por otro lado, el mapeo permitió hacer un cálculo aproximado de la superficie ocupada por cada uno de los tipos de construcciones. Se pudo así llegar a la estimación de las siguientes superficies:

Línea 4 de PROCREAR: promedio de 70 has. producidas en cinco años.

Barrios Cerrados: 680 has. construidas; y 629 has. aprobadas, en venta (producidas desde los años 90 a la actualidad).

Barrios informales: según el Registro Público Provincial de Villas y Asentamientos (RPPVAP), la superficie ocupada por Villas, Asentamientos y Otros en La Plata equivale a $998 \mathrm{Has}$, de los cuales 667 Has fueron producidas durante el periodo 2000-2015. A este dato podamos agregar el de la cantidad de superficie de tierras que intentaron ser ocupadas en los últimos 15 años, equivalente a 590 has. 
A pesar de que el caso de Pro.Cre.Ar abarca menos años que los otros dos casos, y sólo se tiene en cuenta una parte de lo producido por este programa, sí podemos notar que la producción de urbanizaciones cerradas y de barrios presenta dimensiones similares, ambas de gran envergadura. Tenemos presente que las comparaciones no coinciden en recortes temporales, pero el objetivo es poder reconocer la expresión actual de estas formas de producción urbana, más allá de sus temporalidades, y sus estrategias para ser parte de la ciudad.

Podemos decir entonces que el periurbano de la ciudad se fue definiendo en las últimas décadas según las ocupaciones de distintos sectores sociales, lo que resulta en diversas expresiones urbanas, las que reciben distintas respuestas e intervenciones estatales para sus desarrollos.

Veamos ahora las formas de acceso a la tierra. Así como fue descripto, a partir del PROCREAR en el caso de La Plata se desarrollaron 11 barrios producto de procesos colectivos. Estos barrios se dieron lugar en tierras cercanas a zonas urbanizadas, pero de zonificación rural y por tanto sin conexión a servicios básicos y en muchos casos lejanas a infraestructuras básicas de salud, educación, espacios públicos y transporte.

Si bien la ordenanza preveía la intervención estatal en la definición de los precios de suelos, se dieron casos en que el precio fue acordado directamente entre propietarios/as y beneficiarios/as.

Es relevante mencionar la escalada de sobrevaloración de los precios del suelo que se inició ante las explicadas rezonificaciones. Tierras que estaban en desuso, con pago de impuestos de suelo rural (menores a los urbanos), pasaron a valer un $1000 \%$ más a partir del cambio de zonificación. Esta afirmación pudo corroborarse en cinco de los 11 barrios analizados, a partir de relevamientos y entrevistas que arrojaron datos sobre los precios anteriores y posteriores (Tabla 4): 
Tabla 4. Comparación de valores del suelo a partir de la rezonificación de tierras rurales a urbanas y porcentaje de aumento del precio. Análisis en cinco barrios de La Plata surgidos de la vía 2 del 11094/13.

\begin{tabular}{|l|c|c|c|}
\hline Barrio & $\begin{array}{c}\text { Precio del suelo } \\
\text { para zona Rural }\end{array}$ & $\begin{array}{c}\text { Precio del suelo } \\
\text { rezonificado ** }\end{array}$ & \% aumento \\
\hline La Hermosura Ruta 11 y 645 & $\$ 1.955 .000$ & $\$ 19.875 .000$ & $1.016,62$ \\
\cline { 1 - 2 } Bo.Mil Flores. Loteo H - 413 y 136 & $\$ 966.000$ & $\$ 8.892 .000$ & 920,50 \\
\cline { 1 - 2 } El Gigante del Oeste - 47 y 173 & $\$ 5.520 .000$ & $\$ 54.000 .000$ & 978,26 \\
\cline { 1 - 2 } La Esperanza - 610 y 137 & $\$ 1.219 .000$ & $\$ 15.750 .000$ & $1.292,04$ \\
\cline { 1 - 2 } Arturo Seguí 647 y 14bis & $\$ 1.150 .000$ & $\$ 10.780 .000$ & 937,39 \\
\hline
\end{tabular}

* Según el observatorio de precios de suelo (Proyecto H636 SCyT-UNLP), antes del anuncio del

Pro.Cre.Ar. el precio promedio de la tierra mayor a una hectárea en área complementaria o rural en el partido de La Plata rondaba los $23 \$ / \mathrm{m} 2$

** Se estima que del valor que figura en la Tabla, el $35 \%$ del valor fue utilizado para las tareas de urbanización.

Fuente: elaboración propia.

Sobre los asentamientos informales podemos afirmar a partir de la lectura bibliográfica y el trabajo de campo realizado desde organizaciones sociales y proyectos de extensión, que el acceso a la tierra se da por dos vías: ocupación de tierras o mercado informal del suelo. De la primera de las vías, se desprenden gran cantidad de casos en que las tierras ocupadas pasan a formar parte del mercado informal del suelo. La situación de informalidad en los procesos de acceso a estas tierras implica una menor restricción en los requisitos de compra venta. Haciendo una comparativa entre los requisitos para los mercados formal e informal, encontramos que en marcos de informalidad no suelen requerirse situaciones laborales formales, garantías, depósitos (Clichevsky, Cravino). Los procesos informales lo son en sentido amplio: quienes participan en informalidad del mercado de suelo, provienen de situaciones de informalidad laboral, y de familias generalmente ligadas también a otras informalidades. Por tanto, ser parte de la informalidad en cualquiera de sus aspectos no es en general una elección, sino un legado o un resultado de una cadena de procesos.

Las condiciones informales se trasladan a su vez a aspectos ambientales: las tierras ocupadas se caracterizan (siempre en términos generales) (4) por ubicarse cercanas a tierras de 
descarte, tales como bañados, cavas, canteras, arroyos, zonas anegables e inundables. Estas tierras no aptas para ser habitables, no reúnen las condiciones legales ni ambientales para ser incorporadas al mercado formal, son tierras "de descarte", que terminan por ser aprovechadas por los sectores populares. Para el caso de La Plata, cerca del $48 \%$ de sus barrios informales tienen problemas ambientales, referidos a asentarse sobre áreas inundables (al borde de arroyos, sobre camino de sirga, en terrenos bajos o humedales, cerca de canteras, cerca de basurales, sobre suelo degradado), y el $19 \%$ se enfrenta a problemas urbanos (asentados sobre terraplenes ferroviarios, reserva de traza urbana o de electroducto, o de difícil acceso) (RPPVAP, 2015).

Sobre los valores de estos suelos, si bien no se ha trabajado aún en un registro de los precios que se manejan en este mercado, a partir del trabajo de campo se puede presumir que los valores de las tierras son severamente menores en comparación a los precios del mercado formal. En entrevistas en asentamientos y tomas de tierras, se han registrado precios de terrenos de entre un 5 a un $25 \%$ del valor de un lote del mercado formal, pero estos registros no conforman una muestra que pueda considerarse representativa. Sin embargo, podemos decir que el valor del precio es inversamente proporcional a la seguridad en la tenencia de esas tierras.

A pesar de que no desarrollaremos este tema, creemos relevante mencionar que en el mismo Partido donde se hace cada vez más visible la dificultad para acceder al suelo urbano por parte de los sectores populares, se convive con una superficie de tierras vacantes servidas equivalentes a 52.000 lotes de $300 \mathrm{~m} 2$, o 78.000 lotes de $200 \mathrm{~m} 2$ (Frediani, 2016). Si a este valor le agregamos el del precio del suelo, resulta que esos terrenos ya listos para ser habitados tienen un valor fiscal de 183 millones de pesos, y un valor de mercado de 259 millones de dólares (Del Río, 2016). Se hace evidente la disputa de poder que implica la tenencia de tierra.

Para el caso de los barrios cerrados, es posible afirmar (aunque aún no cuantitativamente) que una gran cantidad de estos barrios no se encuentran declarados, por lo que -entre otras cosasaún aportan impuestos por el valor de tierras rurales, o macizos indivisos. El dato de la cantidad exacta de este tipo de barrios no declarados al fisco está aún en construcción, para lo que se realizará una comparativa entre los planos parcelarios de La Plata y el mapa construido para este trabajo. El mismo trabajo se realizará para evaluar las características urbanísticas (accesibilidad, infraestructura y servicios).

Sobre las características de las tierras utilizadas, no toma tanta relevancia las posibilidades de conexión a servicios e infraestructura (pues generalmente tienen posibilidades económicas de ser resueltos por cuenta propia), sino la conectividad de vías de comunicación que presenten. 
Por lo relevado al momento, encontramos que una de las formas más difundidas para acceder a estas tierras es mediante inmobiliarias desarrolladoras de proyectos urbanos y loteos cerrados.

Por último, veamos las características que cada sector social adopta -acorde a sus posibilidades- para el desarrollo urbano de sus barrios. Para realizar una comparativa sobre estos procesos, aclararemos primera y nuevamente la dificultad encontrada, y aún no resuelta en lo que respecta a barrios cerrados. Podemos deducir mediante el análisis bibliográfico que en estos barrios el acceso a servicios e infraestructura se resuelve "puertas adentro", a través de recursos propios, sin necesidad de intervención estatal. Así como hay una intención de autosegregación en términos urbanísticos con pares de la misma clase social, lo mismo se replica en el uso de servicios de salud y educación (haciendo uso de servicios privados propios del sector, accesibles sólo mediante el pago de elevadas cuotas). Respecto del uso de transporte, es también un servicio prescindible, debido al alto uso de automóviles y transporte privado.

Resulta relevante mencionar que diversos autores (principalmente Patricia Pintos) indican que el aumento de la superficie construida de emprendimientos cerrados (y los movimientos de suelos que conllevan, así como los rellenos que se realizan para elevar la cota de inundación), son señalados como factores causantes de las inundaciones que se dieron en La Plata en los últimos años (enero de 2002, marzo de 2005, febrero de 2008, marzo de 2010), algunas de las que tuvieron mayor impacto en el eje noroeste, donde se ubican la mayoría de estos barrios.

Para el caso de los barrios en análisis surgidos del Pro.Cre.Ar., a pesar de que la ordenanza preveía que los/as propietarios/as asumieran los gastos y tareas de luz, agua y apertura de calles, en varios de los barrios estas tareas fueron llevadas adelante por los/as beneficiarios/as. De las 11 nuevas urbanizaciones, se accedió a entrevistar participantes de cinco de ellos, en base a lo que pudimos reconocer los actores que asumieron los costos, así como las estrategias elegidas para acceder a una serie de categorías seleccionadas para caracterizar el desarrollo urbano de estos barrios (Tabla 5): 
Tabla 5. Procesos de desarrollo urbano para barrios PROCREAR en La Plata, surgidos de la vía 2 de la Ordenanza 11094/13

\begin{tabular}{|c|c|c|c|c|c|}
\hline Servicio & $\begin{array}{l}\text { La Hermosura } \\
\text { Ruta } 11 \text { y } 645\end{array}$ & $\begin{array}{llll}\text { Mil } & \text { Flores. } & \text { Loteo } \\
\text { H } & & & \\
413 & \text { a } & 415 & 136 \\
139 & & & \end{array}$ & \begin{tabular}{|l} 
El Gigante del \\
Oeste \\
47 a $52 \quad 173$ a \\
177
\end{tabular} & $\begin{array}{l}\text { La Esperanza } \\
610 \text { y } 137\end{array}$ & $\begin{array}{l}\text { Arturo Seguí } \\
647 \text { a } 650 \text { e } 14 \\
\text { bis y } 16\end{array}$ \\
\hline Luz & $\begin{array}{l}\text { Lo asumió el } \\
\text { vendedor }\end{array}$ & $\begin{array}{l}\text { La gestionaron } \\
\text { los/as } \\
\text { beneficiarios/as }\end{array}$ & $\begin{array}{l}\text { Lo asumió el } \\
\text { vendedor }\end{array}$ & $\begin{array}{ll}\text { Lo asumió el } \\
\text { vendedor pero } \\
\text { se sumó al } \\
\text { precio del } \\
\text { terreno }\end{array}$ & $\begin{array}{l}\text { La gestionaron } \\
\text { los/as } \\
\text { beneficiarios/as }\end{array}$ \\
\hline Agua & $\begin{array}{l}\text { Tienen } \\
\text { perforaciones } \\
\text { que pagó cada } \\
\text { beneficiario/a }\end{array}$ & $\begin{array}{l}\text { Los/as } \\
\text { beneficiarios/as } \\
\text { contrataron una } \\
\text { empresa privada }\end{array}$ & $\begin{array}{l}\text { Los/as } \\
\text { beneficiarios/as } \\
\text { gestionaron con } \\
\text { la Cooperativa } \\
\text { de Olmos }\end{array}$ & $\begin{array}{lr}\text { El vendedor } \\
\text { pagó las } \\
\text { perforaciones }\end{array}$ & $\begin{array}{l}\text { La gestionaron } \\
\text { los/as } \\
\text { beneficiarios/as }\end{array}$ \\
\hline $\begin{array}{l}\text { Apertura } \\
\text { de calles }\end{array}$ & $\begin{array}{l}\text { La gestionaron } \\
\text { los/as } \\
\text { beneficiarios/as }\end{array}$ & $\begin{array}{lr}\text { La } & \text { gestionaron } \\
\text { los/as } & \\
\text { beneficiarios/as } \\
\text { (para } & \text { calle, luz y } \\
\text { agua } & \text { pusieron } \\
\$ 40.000 & \text { cada } \\
\text { familia) } & \end{array}$ & $\begin{array}{l}\text { Lo asumió el } \\
\text { vendedor }\end{array}$ & $\begin{array}{l}\text { Lo asumió el } \\
\text { vendedor pero } \\
\text { se sumó al } \\
\text { precio del } \\
\text { terreno }\end{array}$ & $\begin{array}{l}\text { Lo asumió } \\
\text { vendedor }\end{array}$ \\
\hline Cloaca & No tienen & No se menciona & $\begin{array}{l}\text { Lo hizo cada } \\
\text { beneficiario/a }\end{array}$ & $\begin{array}{l}\text { No tienen } \\
\text { (usan pozo } \\
\text { que se hizo en } \\
\text { cada vivienda) }\end{array}$ & No tienen \\
\hline Gas & No tienen & $\begin{array}{l}\text { Se acordó con la } \\
\text { delegación } \\
\text { municipal, y con un }\end{array}$ & 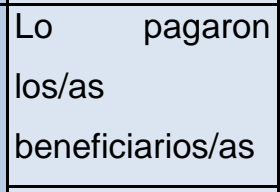 & No tienen & No tienen \\
\hline $\begin{array}{l}\text { Espacio } \\
\text { Público }\end{array}$ & & $\begin{array}{l}\text { proyecto en el } \\
\text { Concejo Dliberante } \\
\text { fueron declarados }\end{array}$ & \begin{tabular}{|l|} 
Definieron entre \\
los/as \\
beneficiarios/as
\end{tabular} & \begin{tabular}{|l} 
Definieron \\
entre los/as \\
beneficiarios/a
\end{tabular} & No tienen \\
\hline
\end{tabular}




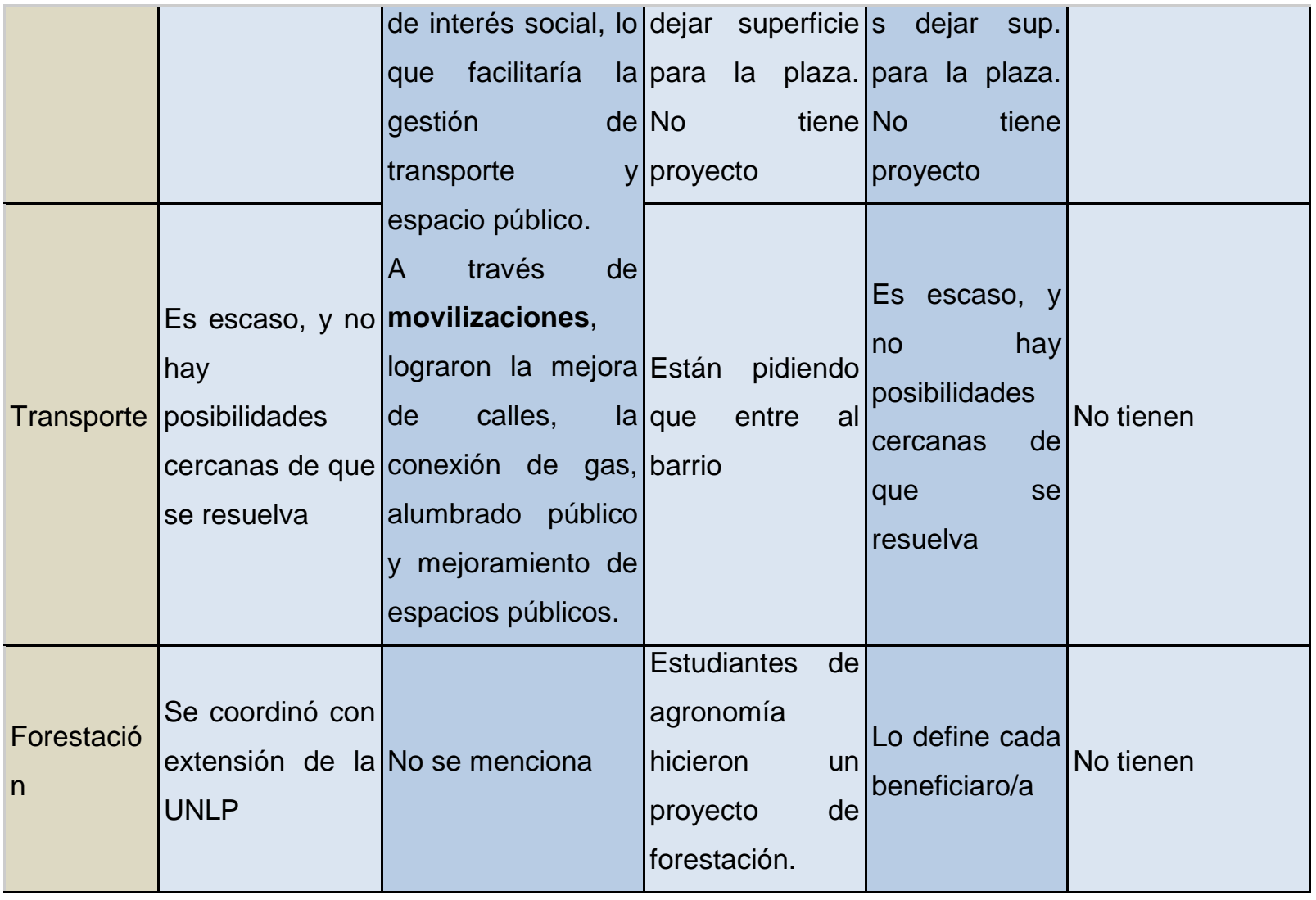

Fuente: elaboración propia a partir de entrevistas.

Este análisis nos permite reconocer por un lado la falta de intervención estatal en los procesos de urbanización. Por otro lado, es notoria la necesaria autogestión y organización vecinal que debió desarrollarse, por lo menos en estos cinco barrios, para poder acceder a servicios básicos. Así como en una primera instancia se requirió de organización para acceder al suelo, el proceso de desarrollo urbano requirió los mismos procesos y esfuerzos. A su vez, podemos reconocer que, a pesar de que la Ordenanza determina las obligaciones para los/as vendedores/as de las tierras, estas no fueron necesariamente cumplidas (aunque el valor de sus tierras -y por tanto sus ganancias- haya sido incrementado en altísimos porcentajes).

Por último, en lo que respecta a los asentamientos informales, el RPPVAP nos confirma la hipótesis de que la localización de estos asentamientos resigna el acceso a servicios básicos, así como cercanía al acceso a servicio de transporte, a los centros urbanos y los bienes que en ellos se emplazan (Gráfico 1):

Gráfico 1. Conectividad a servicios básicos en asentamientos informales de La Plata 


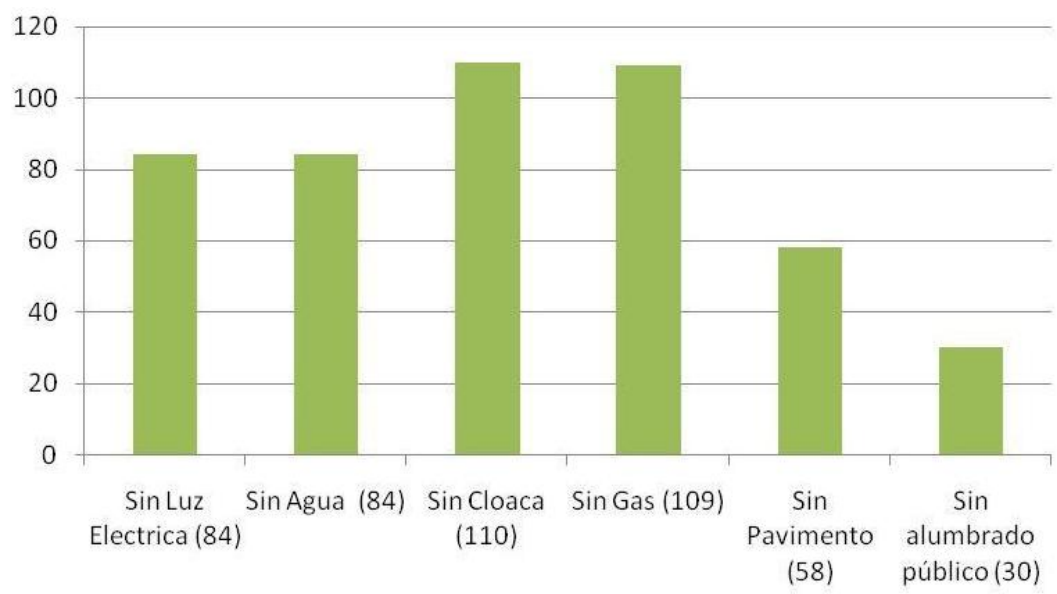

Fuente: elaboración propia a partir del RPPVAP.

El análisis bibliográfico, pero principalmente el trabajo de campo, nos permiten afirmar que las estrategias de este sector para resolver el acceso a servicios básicos se da principalmente mediante la autogestión, más precisamente a través de la auto-conexión a los servicios de formas precarias. Los intentos de acceder a conexiones legales encuentran dos obstáculos: por un lado, muchas empresas prestatarias de servicios locales no conectan al servicio a quien no posee título de propiedad o contrato legal de alquiler. Por otro lado, en los casos en que se logra gestionar, es corriente la dificultad para el pago al servicio, así sea mediante "cuotas sociales", debido a la mencionada cadena de informalidades del que forman los/as habitantes de estos barrios. Las conexiones informales traen aparejados diversos problemas, principalmente el incendio de viviendas por conexiones eléctricas defectuosas; la contaminación del agua para consumo por filtración en las mangueras; contaminación de las napas por pozos ciegos realizados precariamente; entre tantos otros.

Algunos elementos de infraestructura urbana básica son conseguidos en varios casos mediante procesos de exigibilidad, y en muchos casos el mantenimiento cotidiano queda a manos de los/as usuarios/as (como casos de escuelas, salitas de salud, plazas, etcétera). Hemos registrado gran cantidad de barrios que en sus inicios han desarrollado el proceso de apertura de calles por su propia cuenta, "a pala", y con los años (y nuevamente mediante procesos de organización y exigibilidad), lograron que el Estado lo mejore. Lo mismo podemos relatar sobre la recolección de basura e iluminación de las vías públicas.

Podemos en síntesis decir que para los casos de barrios populares, los procesos de desarrollo urbano tienen una primer etapa de autoconstrucción de viviendas y servicios; mediante organización y exigibilidad en algunos casos se logra mejorar estos barrios con el tiempo, pero el mantenimiento cotidiano tiene un fuerte componente autogestivo. 


\title{
Reflexiones finales
}

Hemos intentado recorrer en este trabajo estrategias para acceder al suelo y para desarrollar ciudad en tres experiencias llevadas adelante por distintos sectores sociales. Pudimos a lo largo del trabajo observar que esas estrategias son diferentes según el poder adquisitivo, el capital cultural, y el sector donde se provenga. Las tres experiencias tienen un impacto fuerte en el Partido en distintos sentidos, generando algunos interrogantes sobre la inequidad social reflejada en el territorio. Una de las principales preguntas surgidas de este trabajo refiere a la relación entre territorio y estigmatización. ¿Qué es la informalidad urbana? ¿Por qué cuando se estudia a la informalidad urbana se da lugar mayoritariamente a estudios sobre villas y asentamientos, y no se consideran los barrios cerrados, o algunos casos de la clase media? Rolnik señala que

\begin{abstract}
en realidad, lo que necesitamos es hacer del territorio ciudad, producir ciudad para todos, y no la hemos producido. Esta autora indica que hemos hecho, con la definición de la zonificación para las zonas consolidadas de la ciudad, una reserva de tierra para las clases medias y altas mucho más grande que el tamaño de ese mercado. $Y$ el otro lado de la moneda es que no hemos asignado ubicaciones centrales y consolidadas para los mercados populares del suelo (2008).
\end{abstract}

Las expresiones sociales en el territorio generan certezas sobre la necesidad de promover un modelo urbano con mayor cohesión social en el ámbito urbano (hasta tanto existan las diferencias entre clases).

El modelo de producción urbano se conforma por "piezas", que así como el caleidoscopio, generan movimientos en la totalidad al moverse (Abramo, 2012: 62). Esos movimientos no pueden ya seguir siendo autónomos: sin promover la sobre regulación,

para orientarse en este mar embravecido de la ciudad caleidoscópica (com-fusa) no vale encomendarse al auxilio hipotético de alguna mano invisible caritativa (el mercado): son necesarios timón y brújula, es decir, se requiere de la acción política democrática, de nuevo de la planificación, y de la comprensión en conjunto de la dinámica urbana (Ibídem: 67). 
Notas

(1) Aunque se tiene en cuenta que comparar con distintos recortes temporales no es metodológicamente adecuado, el objetivo del trabajo lo justifica en tanto que se propone evaluar la situación actual de gran parte de la periferia local.

(2) Lectura de imágenes aéreas, revisión de avisos publicitarios, trabajo de campo.

(3) Equipo del que la autora forma parte, aún no publicado. El trabajo desarrolló un Registro de Tomas de Tierras en La Plata en el periodo 2000-2016. El Registro fue desarrollado al momento con fuentes periodísticas, y se encuentra en completamiento con otras fuentes. Se registraron los intentos de ocupaciones, sin tener en valor el fin del proceso.

(4) Decimos términos generales porque tenemos presente que hay tierras que son ocupadas que forman parte del mercado formal de suelo, que son aptas ambientalmente, y que forman parte de otras razones de abandono, tales como herencias vacantes, tierras de engorde, tierras de difícil venta, etcétera.

\section{Bibliografía}

Abramo, P. (2012). La ciudad com-fusa: mercado y producción de la estructura urbana en las grandes metrópolis latinoamericanas. EURE (Santiago), 38(114), pp. 35-69. Recuperado de https://dx.doi.org/10.4067/S0250-71612012000200002

del Cueto, C. y Luzzi, M. (2008). Rompecabezas. Transformaciones en la estructura social argentina (19863-2008). Los Polvorines, Buenos Aires, Argentina: Edit.UNGS.

Del Río, J. P. (2012) El lugar de la vivienda social en la ciudad: Un análisis de la política habitacional desde el mercado de localizaciones intra-urbanas y las trayectorias residenciales de los habitantes. (Tesis de Posgrado). Facultad de Humanidades y Ciencias de la Educación, Universidad Nacional de La Plata. Recuperado de http://www.memoria.fahce.unlp.edu.ar/tesis/te.464/ te.464.pdf

Frediani, J. C. (2009). Las nuevas periferias en el proceso de expansión urbana. Geograficando: Revista de Estudios Geográficos, 5(5), pp. 103-125.

Frediani, J. C. (2010). Lógicas y tendencias de la expansión residencial en áreas periurbanas. El partido de La Plata, Buenos Aires, Argentina, entre 1990 y 2010. (Tesis de Doctorado). Universidad Nacional de La Plata. Recuperado de http://sedici.unlp.edu.ar/handle/10915/3203 
Frediani, J. C. (2016). Mercado de suelo y tierra abandonada. Conferencia en el Curso de Regularización Urbano Dominial y producción del suelo urbano, Presidencia, Universidad Nacional de La Plata.

Garibay, A. (2017). El Pro.Cre.Ar en la ciudad de La Plata: El caso del barrio "Mil Flores". (Tesis de grado). Facultad de Humanidades y Ciencias de la Educación, Universidad Nacional de La Plata. En Memoria Académica. Recuperado de http://www.memoria.fahce.unlp.edu.ar/tesis/te.1336/te.1336.pdf

González, P. (2009). Asentamientos populares en la Gran La Plata: un análisis de las nuevas territorialidades ante las transformaciones del mundo social-comunitario en la década del 2000. En XI Jornadas de Investigación del Centro de Investigaciones Geográficas y del Departamento de Geografía. La Plata. Recuperado de http://www.fuentesmemoria.fahce.unlp.edu.ar/trab eventos/ev.830/ev.830.pdf

Jaramillo, S. (2003). Los fundamentos económicos de la participación en plusvalías. Recuperado de https://bit.ly/1P0twkj

Ventura, V. (2015). La implementación del Pro.Cre.Ar en La Plata. El caso de la ordenanza 11094/13. (Tesis de grado). Facultad de Humanidades y Ciencias de la Educación, Universidad Nacional de La Plata. Recuperado de http://www.memoria.fahce.unlp.edu.ar/tesis/te.1090/te.1090.pdf

Yujnovski, O. (1984). Claves políticas del problema habitacional argentino, 1955-1981. Buenos Aires, Argentina: Grupo Editor Latinoamericano. 